\title{
Apparent superballistic dynamics in one-dimensional random walks with biased detachment
}

\author{
Chapin S. Korosec $\odot,{ }^{*}$ David A. Sivak $\odot,{ }^{\dagger}$ and Nancy R. Forde $\odot{ }^{\ddagger}$ \\ Department of Physics, Simon Fraser University, 8888 University Drive, Burnaby, British Columbia, Canada V5A 1S6
}

(Received 7 May 2020; accepted 24 August 2020; published 29 September 2020; corrected 28 October 2020)

\begin{abstract}
The mean-squared displacement (MSD) is an averaged quantity widely used to assess anomalous diffusion. In many cases, such as molecular motors with finite processivity, the dynamics of the system of interest produce trajectories of varying duration. Here, we explore the effects of finite processivity on different measures of the MSD. We do so by investigating a deceptively simple dynamical system: a one-dimensional random walk (with equidistant jump lengths, symmetric move probabilities, and constant step duration) with an origin-directed detachment bias. By tuning the time dependence of the detachment bias, we find through analytical calculations and trajectory simulations that the system can exhibit a broad range of anomalous diffusion, extending beyond conventional diffusion to superdiffusion and even superballistic motion. We analytically determine that protocols with a time-increasing detachment lead to an ensemble-averaged velocity increasing in time, thereby providing the effective acceleration that is required to push the system above the ballistic threshold. An MSD analysis of burnt-bridges ratchets similarly reveals superballistic behavior. Because superdiffusive MSDs are often used to infer biased, motorlike dynamics, these findings provide a cautionary tale for dynamical interpretation.
\end{abstract}

DOI: 10.1103/PhysRevResearch.2.033520

\section{INTRODUCTION}

The mean-squared displacement (MSD) is often used to assess anomalous diffusion in molecular systems. A system with an MSD that grows linearly in time is conventionally diffusive, lacking anomalous effects [1],

$$
\operatorname{MSD}=\left\langle x^{2}(t)\right\rangle=2 D t .
$$

For processes that obey (1), such as a discrete-time random walk, the displacement distribution after $N$ steps limits to a Gaussian distribution. That is, the $N$ individual steps of a discrete-time random walk are independent and identically distributed, with their sum governed by the central limit theorem [2].

Anomalous diffusion refers to systems that do not have a linear time dependence of the MSD. Generally, anomalous diffusion is thought to emerge in stochastic systems whose displacement distributions are not Gaussian, and is therefore intimately connected with the breakdown of the central limit theorem [2]. The concept of anomalous diffusion was first introduced in 1926 by Richardson [3] through a thought experiment involving two independent air particles separated by a sufficiently large distance so as to be caught by two independent gusts of wind moving in opposite directions. Richardson

\footnotetext{
*ckorosec@sfu.ca

†dsivak@sfu.ca

‡nforde@sfu.ca
}

Published by the American Physical Society under the terms of the Creative Commons Attribution 4.0 International license. Further distribution of this work must maintain attribution to the author(s) and the published article's title, journal citation, and DOI. hypothesized that such a system does not obey Fick's second law, and that the MSD of the air particles scales nonlinearly with time. For an anomalously diffusive system,

$$
\operatorname{MSD} \propto D_{\mathrm{g}} t^{\alpha},
$$

where $D_{\mathrm{g}}$ is the generalized diffusion coefficient, and $\alpha$ is the anomalous diffusion exponent that distinguishes the type of diffusion $[2,4,5]$. Subdiffusion, conventional diffusion, and superdiffusion correspond to $0 \leqslant \alpha<1, \alpha=1$, and $1<\alpha<$ 2 , respectively. The ballistic threshold is at $\alpha=2$, describing a system whose trajectories proceed at constant velocity.

In order for a microscopic system to behave ballistically over long times an external stimulus is typically required. For example, ballistic motion is achieved for random walkers subject to certain forms of external noise [6]. Ballistic motion can also be achieved without an external stimulus in systems whose cooperative behavior limits the degrees of freedom of individual particles [7,8]. To exceed $\alpha=2$, reaching the superballistic regime, it is generally thought an acceleration is required [9]. Examples include animal movement generated by muscle contraction [10] and particles optically trapped in air [11,12], subject to increasing temperatures [13], or subject to expanding media [14].

In this paper we consider a deceptively simple onedimensional discrete random walk with equal-sized and equal-duration steps. We impose no external forces, particle thrust, noise typically thought to promote superdiffusive motion, or cooperative behavior. We instead impose a tunable detachment probability $d$ that produces finite processivity. For every step toward its initial position, the random walker permanently detaches with probability $d$. We explore two detachment protocols: constant detachment, and detachment exponentially increasing at rate $k_{\mathrm{d}}$. 
TABLE I. Probability distributions for the first four time steps of the ensemble-level model.

\begin{tabular}{lccccccccr}
\hline \hline Time & \multicolumn{10}{c}{ Displacement } \\
\cline { 2 - 10 } & -4 & -3 & -2 & -1 & 0 & 1 & 2 & 3 & 4 \\
\hline 0 & & & & & 1 & & & & \\
1 & & & & $\frac{1}{2}$ & 0 & $\frac{1}{2}$ & & & \\
2 & & & $\frac{1}{4}$ & 0 & $\frac{1}{2} r$ & 0 & $\frac{1}{4}$ & & \\
3 & $\frac{1}{8}$ & 0 & $\frac{3}{8} r$ & 0 & $\frac{3}{8} r$ & 0 & $\frac{1}{8}$ & \\
4 & $\frac{1}{16}$ & 0 & $\frac{1}{4} r$ & 0 & $\frac{3}{8} r^{2}$ & 0 & $\frac{1}{4} r$ & 0 & $\frac{1}{16}$ \\
\hline \hline
\end{tabular}

By varying $d$ or $k_{\mathrm{d}}$, we find that the anomalous diffusion exponent $\alpha$ can be tuned over a wide range of values. Varying $d$ controls apparent time-dependent dynamics ranging from diffusive to ballistic, while variations in $k_{\mathrm{d}}$ tune $\alpha$ from the diffusive into a transient but long-lasting superballistic regime. We determine the mechanism for this apparently superballistic behavior by analytically deriving the ensemble velocity and find it is dependent on the detachment protocol used, thereby producing the ensemble acceleration required to breach the ballistic threshold. We reproduce several of these observations in a trajectory-resolved model, though find that distinct approaches to calculating the MSD provide different values of $\alpha$ and hence distinct inferences about the system's dynamics. We additionally show that selecting a subset of especially processive trajectories - as is common in single-particle studies [15-17] - can introduce a large superdiffusive bias into the MSD. As an example, we demonstrate that this acceleration by detachment can manifest in a more realistic system by calculating MSD for burnt-bridges ratchets, which we find to exhibit apparently superballistic behavior. Our results encourage increased transparency regarding selection methods for single-particle analysis.

\section{MODELING RANDOM WALKS WITH DETACHMENT}

\section{A. Ensemble-level model}

First, we study a discrete-time diffusion scheme where we track the probability flow of an ensemble of independent onedimensional random walkers. At time intervals $\Delta t=1$, each walker moves a distance $\Delta x=1$, with equal probability of going left or right. A step towards the initial position at the origin incurs a probability of detachment, either with constant or with exponential probability. For constant detachment, the probability $d$ of detaching is independent of time. Exponential detachment probability is given by

$$
d(t)=1-e^{-k_{\mathrm{d}} t},
$$

increasing over time as determined by the rate $k_{\mathrm{d}}$. [Thus the probability of remaining attached at each origin-directed step is $r(t)=1-d(t)$.] Detachment events of individual walkers are independent. Table I shows such a system's initial evolution. For the results shown in this work, the probability distribution was evolved for $n=1000$ time steps.

\section{B. Trajectory-resolved model}

We model a one-dimensional discrete-time random walk by sampling step lengths from a Gaussian distribution with zero mean and unit standard deviation. Steps toward the origin lead to detachment with probability described by (3). As a control, we compare to a random walk with no detachment, which produces conventional diffusion.

\section{ANALYSIS METHODS}

\section{A. Mean-squared displacement and $\alpha(t)$ for ensemble-level model}

We define the MSD for the ensemble-level model as

$$
\operatorname{MSD}_{\mathrm{EA}}=\left\langle x^{2}\right\rangle_{\mathrm{rem}} \equiv \frac{\sum p_{i} x_{i}^{2}}{P_{\mathrm{rem}}},
$$

where $x_{i}$ is the $i$ th position, $p_{i}$ is the probability of having a walker at the $i$ th site, and $P_{\text {rem }}$ is the total remaining probability. $\mathrm{MSD}_{\mathrm{EA}}$ denotes an ensemble-averaged MSD, discussed more in the following section.

The time dependence of the anomalous diffusion exponent $\alpha$ is sometimes calculated through a quantity called either the dynamic functional [18] or the local MSD scaling exponent [19], which is the derivative of the logarithm of the MSD with respect to the logarithm of time [20],

$$
\alpha_{\psi} \equiv \frac{d(\log \mathrm{MSD})}{d(\log \Delta t)} .
$$

It has been noted that the numerical derivative of (5) can become especially noisy at long times because of compression of linear sampling by logarithmic scaling, thereby introducing uncertainty into estimates of the time dependence of $\alpha$ [21].

Alternatively, we introduce a two-point estimator $\alpha_{n-b}^{n}$ for $\alpha$ using time points $n$ and $n-b$. In one dimension and for no average drift $(\langle x\rangle=0)$,

$$
\alpha_{n-b}^{n} \equiv \log _{n}\left(\frac{\left\langle x^{2}(n)\right\rangle}{\left\langle x^{2}(n-b)\right\rangle}\right)\left(1-\frac{1}{\log _{n-b} n}\right)^{-1} .
$$

(Appendix A provides a general derivation.) To the best of our knowledge, (6) has not been used before, and we find it useful for characterizing transient anomalous diffusion with the MSD. It is important to note that $b$ must be kept small in order to extract information about the local slope; for this reason we use $b=2$ in this work when estimating the time dependence of $\alpha$ with (6).

As an example of its utility, in Appendix B we compare $\alpha_{n-b}^{n}$ to $\alpha_{\psi}$ when the MSD undergoes an abrupt change in slope. Figure 8 shows that the numerical derivative from (5) produces a noisy approximation to $\alpha_{\psi}$, whereas $\alpha_{n-b}^{n}$ (6) is smooth throughout.

Table II in Appendix C presents $P_{\text {rem }}, \sum p_{i} x_{i}^{2},\left\langle x^{2}\right\rangle_{\text {rem }}$, and $\alpha_{n-2}^{n}$ for the first four time steps.

\section{B. Mean-squared displacement and $\alpha(t)$ for trajectory-resolved model}

There are many ways to compute the MSD of trajectories; we start by defining the squared displacement as

$$
\Delta x_{j}^{2}(t, \tau) \equiv\left[x_{j}(t+\tau)-x_{j}(t)\right]^{2},
$$


where $x_{j}$ denotes the position of the $j$ th trajectory, $t$ is time, and $\tau$ is the time lag. The mean-squared displacement for an ensemble of $N$ independent walkers is

$$
\left\langle\Delta x^{2}(t, \tau)\right\rangle \equiv \frac{1}{N} \sum_{j=1}^{N} \Delta x_{j}^{2}(t, \tau) .
$$

We define the ensemble-averaged (EA) MSD as the MSD at time $\tau$, relative to the initial time zero,

$$
\operatorname{MSD}_{\mathrm{EA}} \equiv\left\langle\Delta x^{2}(0, \tau)\right\rangle=\frac{1}{N} \sum_{j=1}^{N} \Delta x_{j}^{2}(0, \tau) .
$$

This is equivalent to (4), above. By contrast, the trajectoryaveraged (TA) MSD is computed independently for each trajectory,

$$
\operatorname{MSD}_{\mathrm{TA}} \equiv \overline{\Delta x_{j}^{2}(\tau)}=\frac{\Delta t}{T_{j}-\tau+\Delta t} \sum_{t=0}^{T_{j}-\tau} \Delta x_{j}^{2}(t, \tau),
$$

where $T_{j}$ is the total duration of the $j$ th trajectory, and $\Delta t=1$ is the time step.

The van Hove correlation function or displacement distribution $G(x, \tau)$ collects all the displacements for a particular time lag $\tau$ across all the observed trajectories [19,22-24]. $G(x, \tau)$ describes the probability of a particular displacement for a time lag $\tau$. The variance of $G(x, \tau)$ is the ensemble $\mathrm{MSD}_{\mathrm{TA}}$ (i.e., the $\mathrm{MSD}_{\mathrm{ETA}}$ ) for $\tau$,

$$
\operatorname{MSD}_{\mathrm{ETA}} \equiv\left\langle\overline{\Delta x^{2}(\tau)}\right\rangle=\frac{1}{N} \sum_{j=1}^{N} \overline{\Delta x_{j}^{2}(\tau)}
$$

The MSD $\mathrm{ETA}_{\mathrm{A}}$ is particularly useful for many independent but short trajectories.

MSDs calculated from Eqs. (9)-(11) are asymptotically equal for an ergodic process: The time average converges to the ensemble average at a sufficiently long time. By contrast, differing MSDs may imply nonergodicity; examples of such systems include tracer particles in mucus [25] or the plasma membrane [26], as well as the continuous-time random walk with diverging average waiting times [27].

Here, we estimate $\alpha$ through a linear fit of $\log$ MSD as a function of log time. For visual aid we fit over local regions of approximate linearity and report the range of times used in the fit. Because this logarithmic scaling produces a non-normal distribution of errors, we use a generalized leastsquares (GLS) approach to estimate $\alpha_{\mathrm{GLS}}$ from the MSD [28], employing the function $g l s$ from the $\mathbf{R}$ library nlme.

\section{Shape of probability distributions}

Deviation of the displacement distribution $G(x, \tau)$ from a Gaussian can be quantified by the standardized fourth moment (the kurtosis),

$$
\beta_{2}(x) \equiv \frac{\left\langle(x-\langle x\rangle)^{4}\right\rangle}{\left(\left\langle(x-\langle x\rangle)^{2}\right\rangle\right)^{2}} .
$$
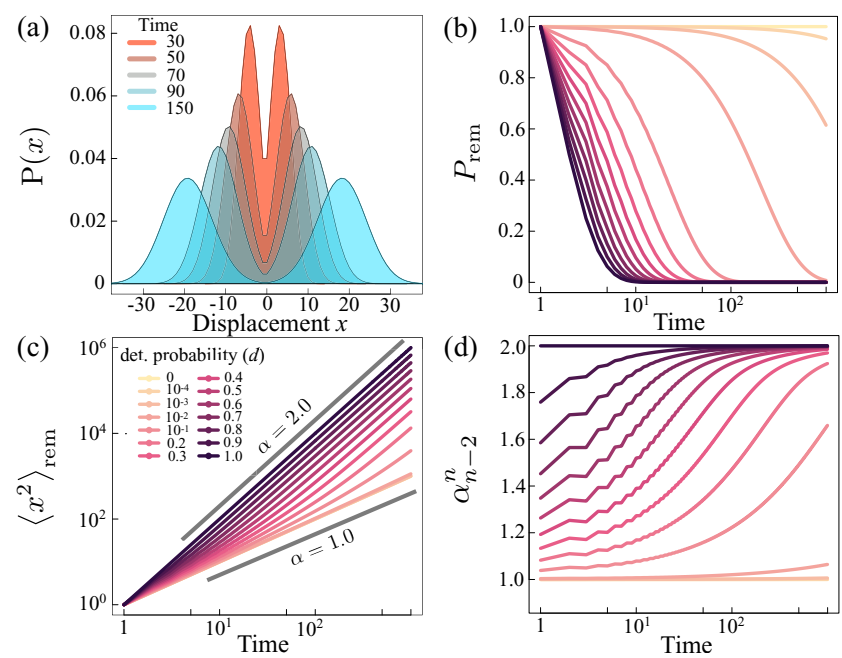

FIG. 1. Ensemble-level analysis with constant detachment (a) Evolution of the displacement distribution for constantdetachment probability $d=0.4$. (b) Probability of remaining on the track as a function of time, for different detachment probabilities $d$. (c) $\mathrm{MSD}_{\mathrm{EA}}$ (4) as a function of time. (d) Diffusion exponent $\alpha_{n-b}^{n}(6)$ as a function of time. For $d=0$ (no detachment), $\alpha_{n-2}^{n}=1$ (conventional diffusion) at all times. As the detachment probability increases to $d=1, \alpha_{n-2}^{n}$ increases to the ballistic limit of 2 .

As a Gaussian distribution has $\beta_{2}(x)=3$, it is convenient to define the excess kurtosis $\gamma_{2} \equiv \beta_{2}-3$, such that any nonzero $\gamma_{2}$ indicates non-Gaussian behavior [29,30].

\section{RESULTS AND DISCUSSION}

\section{A. Ensemble-level analysis}

We first determine the effects of a constant origin-directed detachment bias on the ensemble-level system. Figure 1(a) displays the evolution of the displacement distribution for the ensemble-level analysis with constant-detachment probability $d=0.4$. Two peaks form, symmetric about the origin, and move with increasing dispersion in the $\pm \hat{x}$ directions, respectively. This is expected, as steps towards the origin have a probability of detachment, whereas steps away from the origin do not. Figure 1(b) shows that as the probability $d$ of detachment increases, the probability of remaining on the track decays more rapidly. The $\mathrm{MSD}_{\mathrm{EA}}$ (4) varies with $d$, ranging from a ballistic trend $(\alpha=2)$ for detachment probability $d=1$ to conventional diffusion $(\alpha=1)$ for the no-detachment scenario $(d=0)$ [Fig. 1(c)]. For intermediate $d(0<d<1)$, the MSD displays increasing superdiffusive trends with increasing $d$.

To more quantitatively extract the time dependence of the anomalous diffusion exponent, we calculate $\alpha_{n-2}^{n}$ using (6). For all intermediate values of $d$, Fig. 1(d) shows that $\alpha$ increases with time monotonically towards the ballistic threshold; the transition to the ballistic threshold occurs sooner as $d \rightarrow 1$.

Figure 2 shows the ensemble-level analysis with an exponential time-dependent detachment probability given by (3). $d(t)$ is still bounded by $[0,1]$, but increases monotonically with time towards unity at a rate governed by $k_{\mathrm{d}}$ [Fig. 2(a)]. 

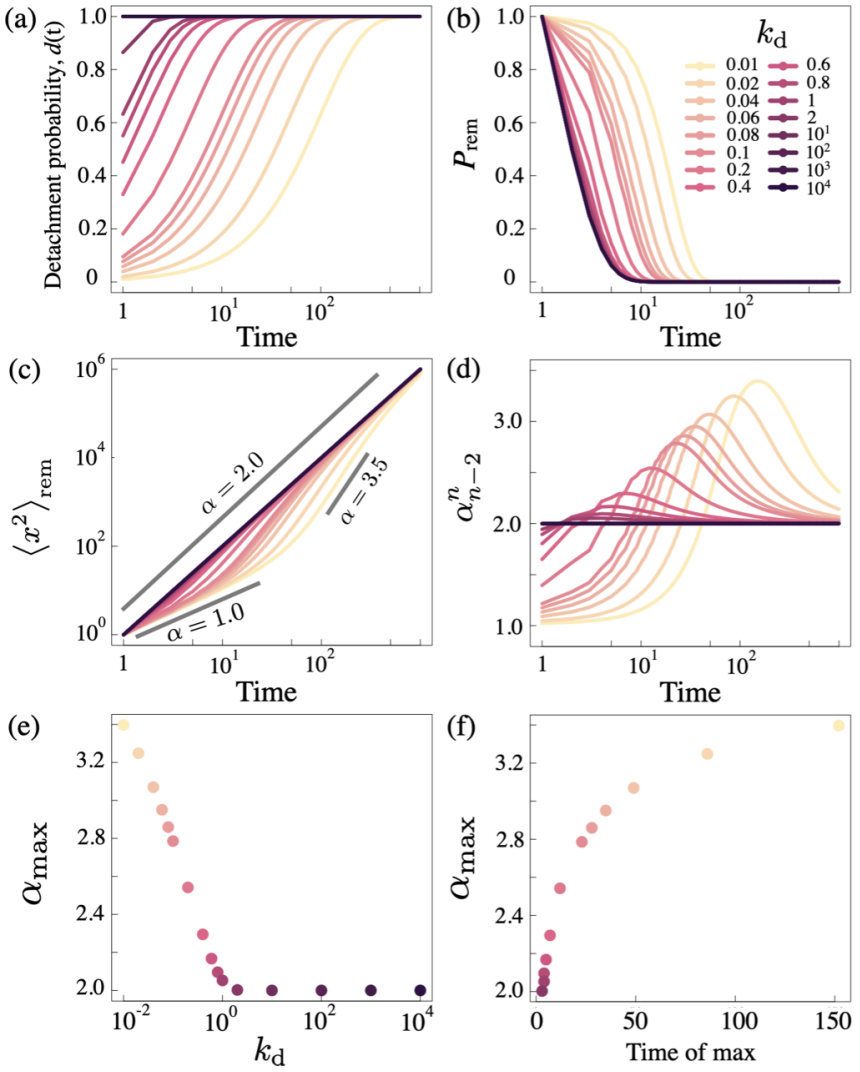

FIG. 2. Ensemble-level analysis with exponential detachment. (a) Time-dependent detachment probability (3) as a function of time for various decay rates $k_{\mathrm{d}}$. (b) Probability of remaining as a function of time for various $k_{\mathrm{d}}$. (c) $\mathrm{MSD}_{\mathrm{EA}}$ (4) as a function of time, for various $k_{\mathrm{d}}$. (d) Transient behavior of $\alpha_{n-2}^{n}$ (6) as a function of time, for various $k_{\mathrm{d}}$. (e) Maximum diffusion exponent $\alpha_{\max }$ as a function of $k_{\mathrm{d}}$. (f) Parametric plot of $\alpha_{\max }$ vs the time at which $\alpha_{\max }$ occurs.

The slowest detachment process studied, corresponding to $k_{\mathrm{d}}=10^{-2}$, leads to the slowest decay of $P_{\text {rem }}$ from 1 to 0 [Fig. 2(b)]. For the fastest detachment process studied $\left(k_{\mathrm{d}}=\right.$ $10^{4}$ ), the $\operatorname{MSD}_{\mathrm{EA}}$ (4) appears ballistic at all times [Fig. 2(c)]. For all other $k_{\mathrm{d}}$ values, we find the MSD to undergo a timedependent transition from diffusive to ballistic, exhibiting a long superballistic transient at intermediate times (peaking at $\alpha \approx 3.5$ for $k_{\mathrm{d}}=10^{-2}$ ). We find that all $k_{\mathrm{d}}<1$ lead to $\alpha_{n-2}^{n}$ rising past the ballistic threshold. This behavior is characterized in Fig. 2(d), in which it is clear that as $k_{\mathrm{d}}$ decreases, the maximum $\alpha_{n-2}^{n}\left(\alpha_{\max }\right)$ increases further into the superballistic regime. Figure 2(e) shows that $\alpha_{\max }$ decreases from strongly superballistic as $k_{\mathrm{d}}$ increases, until for $k_{\mathrm{d}}>1, \alpha_{n-b}^{n}$ reaches and remains near the ballistic limit. Figure 2(f) shows that $\alpha_{\max }$ occurs later as $k_{\mathrm{d}}$ decreases. Therefore, for slower origin-biased detachment (lower $k_{\mathrm{d}}$ ), the system takes longer to breach the ballistic threshold, but extends farther into the superballistic regime and stays superballistic for longer.

When an ensemble of particles moves at the ballistic threshold, their velocity is constant; exceeding the ballistic threshold requires an ensemble acceleration $[\langle a(t)\rangle>0]$. In Appendix D we derive a general expression for the mean velocity $\left\langle v_{+}(t)\right\rangle$ of the positive half of the displacement distribution [see Fig. 1(a)]. For the constant and exponential
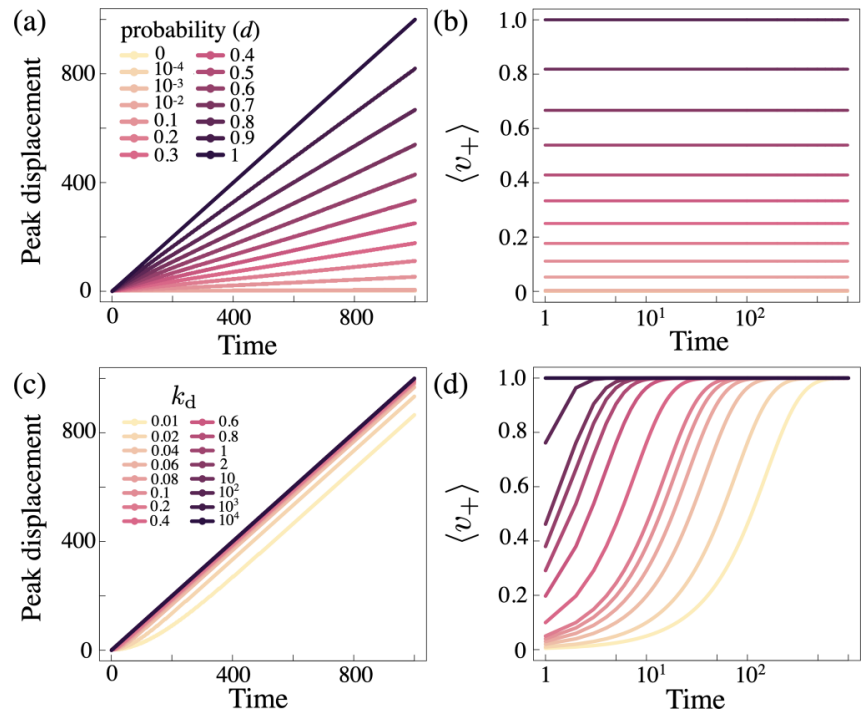

FIG. 3. Ensemble-level analysis of positive peak displacement. (a) Positive peak displacement and (b) its velocity (13), as a function of time for constant detachment. (c) Positive peak displacement and (d) its velocity (14) as a function of time for exponential detachment.

biased-detachment models,

$$
\begin{gathered}
\left\langle v_{+}\right\rangle_{\text {const }}=\frac{d}{2-d}, \\
\left\langle v_{+}\right\rangle_{\exp }=\frac{1-e^{-k_{\mathrm{d}} t}}{1+e^{-k_{\mathrm{d}} t}} .
\end{gathered}
$$

Equation (13) predicts constant velocity (no acceleration; ballistic motion) for constant-detachment probability $(d>0)$, while (14) predicts acceleration (superballistic motion) when the origin-biased detachment probability increases with time according to (3).

We compare these predictions with the dynamics of the ensemble-level displacement distributions (Figs. 1 and 2). For all constant-detachment probabilities, the positive peak displacement linearly increases with time [Fig. 3(b)]. The positive peak displacement for exponential detachment probability [Fig. 3(c)] increases nonlinearly, with velocity given by (14). In Appendix D, we validate (14) by integrating it with respect to time to get the conditional mean displacement (D6) and show that the analytical result agrees with the simulations (Fig. 9).

Despite our system moving with equidistant jump lengths, with equal probability in either direction, and at constant time intervals, the detachment protocol alone is sufficient to push the anomalous diffusion exponent far into the superballistic regime for hundreds of time steps. Therefore, despite no individual particle experiencing acceleration, the selective bias of the detachment protocol produces an ensemble acceleration sufficiently strong to push the diffusion exponent far into the superballistic regime. Biased detachment represents a distinct mechanism to produce apparently anomalous diffusion.

\section{B. Trajectory-resolved analysis}

We have shown in an analytically tractable ensemblelevel analysis that biased detachment is sufficient to tune 

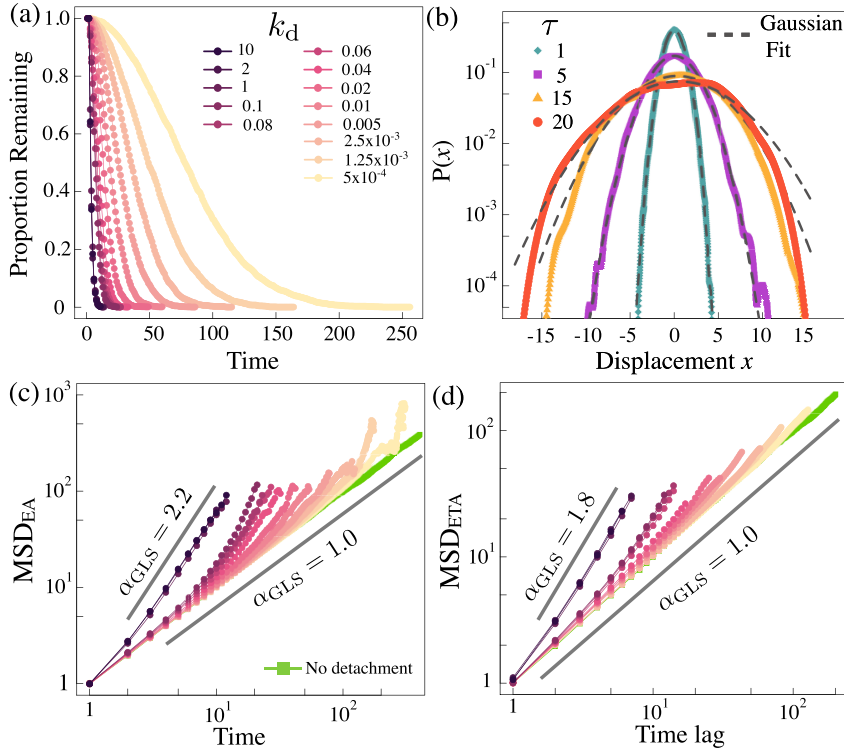

FIG. 4. Trajectory-resolved analysis. (a) Proportion remaining as a function of time for various values of $k_{\mathrm{d}}$. (b) Example Gaussian fits to the displacement distribution for $k_{\mathrm{d}}=0.02$ and time lags $\tau=1,5,15,20$. (c) $\operatorname{MSD}_{\mathrm{EA}}$ (9) as a function of time for each $k_{\mathrm{d}}$. (d) $\operatorname{MSD}_{\mathrm{ETA}}$ (11) as a function of time for each $k_{\mathrm{d}}$.

the anomalous diffusion exponent far into the superballistic regime. Next, we explore the effects of the biased exponential detachment probability (3) in a trajectory-resolved analysis.

For each $k_{\mathrm{d}}$, we simulate $5 \times 10^{4}$ independent trajectories. Figure 4(a) displays the proportion of trajectories still continuing at different times: As $k_{\mathrm{d}}$ increases, detachment happens more rapidly.

We use the Gaussian parameter $\gamma_{2}$ (12) to determine if the displacement distribution conforms to a Gaussian, which is thought to be a requirement for conventionally diffusive systems [2]. For larger time lags, the displacement distribution is non-Gaussian [Fig. 4(b)], reflecting the increased bias as seen in the larger anomalous diffusion exponent $\alpha$. Figure 10 in Appendix E plots $\gamma_{2}$ for various $k_{\mathrm{d}}$ across a large range of time lags, revealing a general trend for negative $\gamma_{2}$ at larger time lags and larger $k_{\mathrm{d}}$.

Figures 4(c) and 4(d) show the MSDEA (9) and the $\operatorname{MSD}_{\mathrm{ETA}}(11)$ for all $k_{\mathrm{d}}$. The MSDEA produces higher $\alpha_{\mathrm{GLS}}$ for every $k_{\mathrm{d}}$, with a maximum $\alpha_{\mathrm{GLS}}=2.2$ for $k_{\mathrm{d}}=10$, compared to 1.8 from the MSDETA with the same $k_{\mathrm{d}}$. Whereas the MSD $_{\text {EA }}$ breaks through the ballistic threshold, the MSD $\mathrm{ETA}_{\mathrm{E}}$ remains in the superdiffusive (sub-ballistic) regime.

For the trajectory-resolved analysis the highest $k_{\mathrm{d}}$ we explore is 10 , where $\alpha_{\mathrm{GLS}}$ peaks at 2.2 and 1.8 for the $\mathrm{MSD}_{\mathrm{EA}}$ and $\mathrm{MSD}_{\mathrm{ETA}}$, respectively [Figs. 4(c) and 4(d)]. $\alpha_{\mathrm{GLS}}$ corresponding to $\mathrm{MSD}_{\mathrm{EA}}$ for the lowest $k_{\mathrm{d}}=5 \times 10^{-4}$ barely increases above the diffusive (no-detachment) limit [Fig. 4(c)]. This appears to be the opposite trend to that seen for the ensemble-level analysis, where the highest values of $\alpha$ occur for the smallest $k_{\mathrm{d}}$ values [Fig. 2(c)]. However, we note that the trajectory-resolved approach explores significantly shorter timescales than the ensemble-level analysis, a result of (in some cases, rapid) detachment of walkers from the track and thus poor statistics at longer times. The early-time

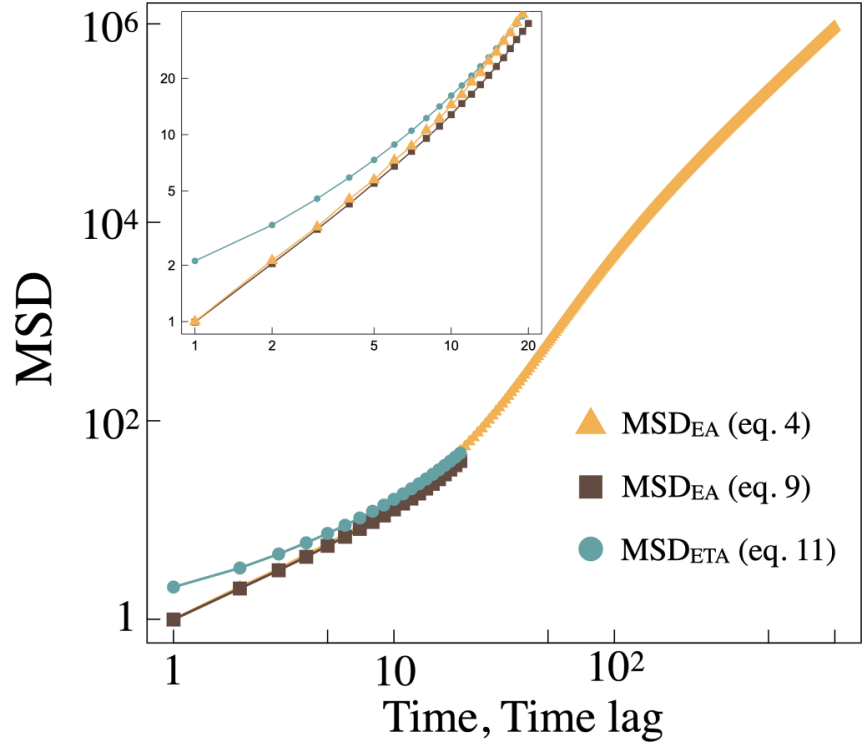

FIG. 5. Trajectory-resolved MSD ETA (11) (blue circles) and $\mathrm{MSD}_{\mathrm{EA}}$ (9) (brown squares) compared with ensemble-level MSD $\mathrm{EA}_{\mathrm{E}}$ (4)(orange triangles), for $k_{\mathrm{d}}=0.04$ and 500000 trajectories. Inset: All MSDs at short times or time lags.

MSD and $\alpha$ do agree between the analytical and numerical approaches [Figs. 2(c) and 4(c)], as we now show quantitatively.

To improve statistical significance, we simulate significantly more $\left(5 \times 10^{5}\right)$ trajectories for $k_{\mathrm{d}}=0.04$. Figure 5 shows trajectory-resolved $\mathrm{MSD}_{\mathrm{EA}}$ (9) and $\mathrm{MSD}_{\mathrm{ETA}}$ (11), alongside the ensemble-level $\mathrm{MSD}_{\mathrm{EA}}$. This comparison makes clear that all MSDs follow the same trend. It also highlights the statistical challenge of achieving superballistic behavior by biased detachment; however, even a small detachment bias $\left(k_{\mathrm{d}}=0.04\right)$ produces significant superdiffusion.

\section{Analysis of longest-duration trajectories}

Studies of molecular-motor transport commonly include in an MSD analysis only a subset of the recorded trajectories, often chosen because they remained associated to the track beyond a threshold duration [15-17] or reached a certain distance [31]. Here, we show that with biased detachment, selecting a longest-lived subset of the total ensemble biases the MSD analysis.

We simulate $2 \times 10^{9}$ independent trajectories with $k_{\mathrm{d}}=0.05$, but only analyze subsets $M$ of these trajectories, selecting the $M_{1}=10^{2}, \quad M_{2}=10^{4}$, and $M_{3}=10^{6}$ longest-duration trajectories for each subset (corresponding to proportions $5 \times 10^{-8}, 5 \times 10^{-6}$, and $5 \times 10^{-4}$, respectively). Figure 6(a) shows the detachment characteristics of these subsets: Each $M$ subensemble experiences a lag time before detachment begins. As the subset size decreases, detachment begins later. Figure 6(b) shows randomly selected trajectories from the longest-duration $M_{1}$ ensemble. Despite their clear stochasticity, they exhibit net motion away from the origin (as expected for these trajectories that avoided early origin-biased detachment).

Figure 6(c) displays $\mathrm{MSD}_{\mathrm{EA}}$ as a function of time for all three $M$ ensembles. At the earliest times the diffusion exponent $\alpha_{\mathrm{GLS}}=1.04$, slightly exceeding conventional diffusion. 

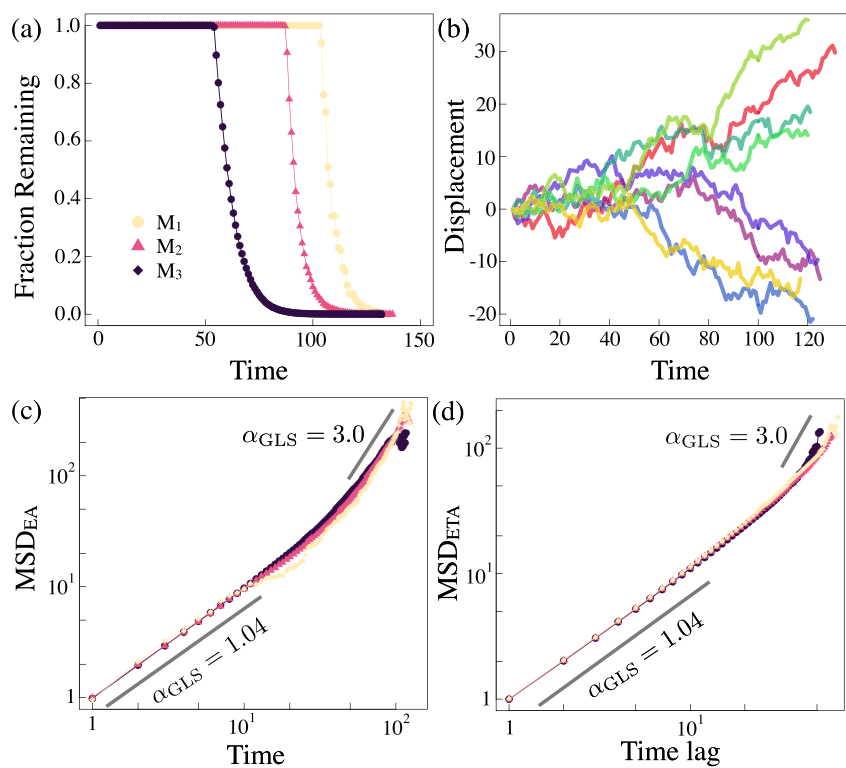

FIG. 6. Longest-duration trajectory-resolved analysis. (a) Fraction remaining as a function of time for the $M$ longest-lived trajectories out of the total ensemble size of $2 \times 10^{9}$. (b) Randomly selected trajectories from the longest-lived $M_{1}$ ensemble. (c) MSD $\mathrm{EA}_{\mathrm{E}}$ (9) and (d) $\operatorname{MSD}_{\mathrm{ETA}}$ (11) as a function of time for all $M$ ensembles.

To more fully explore the effect of processivity, we fit over the full range of timescales for which all trajectories in each of the $M$ ensembles remain attached. We find $\alpha$ of $1.36,1.32$, and 1.25 for $M_{1}, M_{2}$, and $M_{3}$, respectively. Therefore, the size of the subset $M$ influences the reported $\alpha$ even for that subset of trajectories that are fully processive. After the onset of detachment, $\alpha_{\mathrm{GLS}}$ increases far into the superballistic regime and peaks at 3.0, as measured by a generalized least-squares fit of the $M_{1}$ ensemble over the time interval indicated by the black slanted line in Fig. 6(c). The MSD ${ }_{\mathrm{ETA}}$ displays similar trends to the $\mathrm{MSD}_{\mathrm{EA}}$ at both short and long time lags, curving upwards to a steep slope of $\alpha_{\mathrm{GLS}}=3.0$ at long time lags [Fig. 6(d)].

The longest-lived trajectories are those that have managed to escape the "detachment trap" by successively moving away from the origin. That is, although the motion is random, from a sufficiently large ensemble there will be walkers that appear superdiffusive. It is therefore expected that the longest-lived ensembles exhibit superdiffusive characteristics even before the onset of detachment, as shown in Figs. 6(c) and 6(d). These results suggest that selecting a subset of trajectories based on processivity has the potential to strongly bias inferences about the system dynamics, whether one uses the $\mathrm{MSD}_{\mathrm{EA}}$ or MSD $\mathrm{ETA}_{\mathrm{ET}}$. At long times both MSD measures display similar superballistic measures of $\alpha$.

\section{Application to burnt-bridges ratchets}

Some synthetic biomolecular systems, such as molecular spiders [32,33], burnt-bridges ratchets [34], and DNA nanomotors [35-37], remodel their tracks as they move and have been engineered to achieve directional motion at the molecular level. This remodeling turns a substrate site into a product site, and where there is a greater affinity to bind

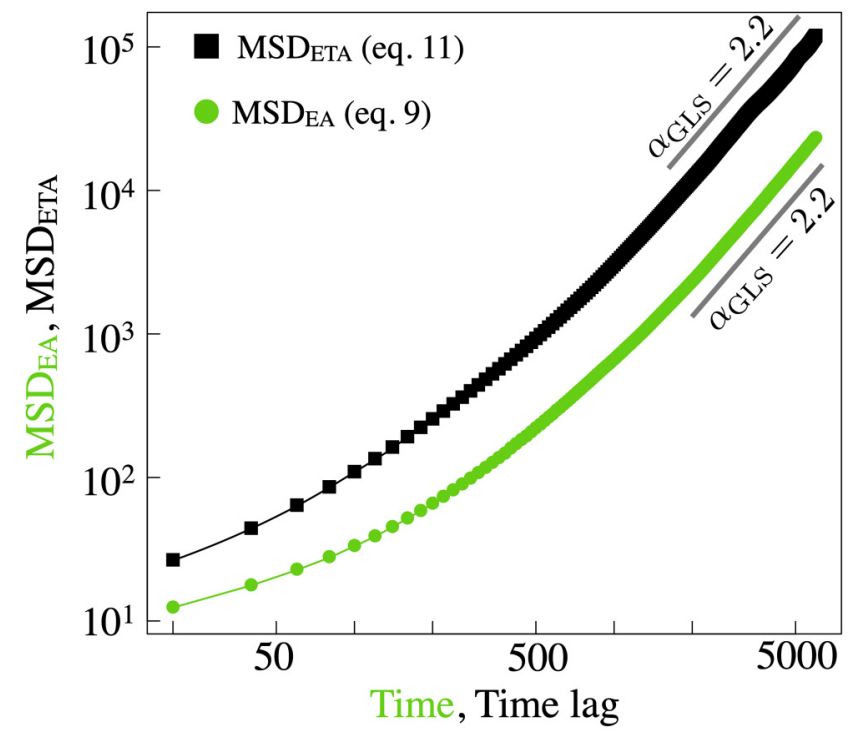

FIG. 7. $M S D_{\mathrm{EA}}$ as a function of time (green) and $\mathrm{MSD}_{\mathrm{ETA}}$ as a function of time lag (black) for burnt-bridges ratchets with finite processivity [41].

to the substrate, motion is biased away from the product wake. Therefore, such systems have an increased probability of detachment from their tracks if they move backwards (into their product wake) [38-41]. Our system is not unlike that of a simple Brownian ratchet [42], traversing a series of boundaries that can be freely passed from the left, but are difficult to cross from the right. In our work we allow movement to occur without inhibition away from the origin, but ascribe a detachment penalty to moves taken towards the origin. Tuning this kinetic penalty leads to an ensemble acceleration that manifests in a highly anomalous diffusion exponent.

To demonstrate that the anomalous $\alpha$ shown above is observed in more realistic systems with finite processivity, we examine simulated trajectories of a burnt-bridges ratchet (BBR), reported previously [41]. Here, we examine an ensemble of 10000 independent BBRs each moving on a quasi-onedimensional (1D) track that is four lattice sites wide. Each BBR has three catalytic legs with a span of eight lattice sites, which can each interact with substrate sites but not product sites. Figure 7 shows that the MSD $\mathrm{EA}_{\mathrm{EA}}(9)$ and $\mathrm{MSD}_{\mathrm{ETA}}$ (11) are quantitatively different. This is not surprising, because initial symmetry breaking leads to distinct short-time and long-time dynamics. In the long-time limit, both the $\mathrm{MSD}_{\mathrm{EA}}$ (9) and $\mathrm{MSD}_{\mathrm{ETA}}$ (11) produce superballistic $\alpha_{\mathrm{GLS}}$ (2.19 and 2.17 , respectively). As no acceleration is imposed on the system, the breach above the ballistic threshold likely arises, as above, from the origin-directed detachment bias inherent to the BBR dynamics. We note that this apparent superballistic behavior is for a specific example of BBRs described in Ref. [41] and may not be a general result for all BBR systems.

\section{IMPLICATIONS}

\section{A. Biological molecular motors}

MSD analysis is often utilized in investigations of intracellular active transport [43]. It has been applied to molecular 
motors such as kinesin [44], dynein [45], and myosin [46]. Of relevance to this work, all of these motors exhibit finite processivity: No molecular motor remains bound to its track forever. This finite processivity was the inspiration for the longest-lived analysis of Sec. IV C: Could duration-based selection of trajectories alter the conclusions drawn from MSD analysis? For example, when calculating the $\mathrm{MSD}_{\mathrm{TA}}$ (10) from experimental data it is common to include only those motors that remained bound to the track for a certain time [15-17], thereby ignoring those that detached. The selection of trajectories for MSD analysis is not always described, which makes it difficult for the interested reader to assess any bias that may manifest in the MSD. (We expect ensemble selection is not typically described in detail because the resulting potential bias in the MSD has yet to be raised.) To our knowledge experimental studies that examine how the MSD changes as a function of the duration of the trajectories chosen are lacking. Such subsampling would demonstrate the robustness of the conclusions drawn from the MSD, and could potentially provide mechanistic insight about detachment kinetics. For example, subsampling may indicate a directional detachment bias, as suggested by the work presented in this paper.

\section{B. Random walks with finite processivity}

Much attention has focused on understanding systems displaying anomalous diffusion. Anomalous diffusion is often correlated with non-Gaussian displacement distributions $[47,48]$. When simulating individual trajectories, our displacements are drawn from a Gaussian distribution; however, our displacement distributions for large time lags break Gaussianity because of the penalty we ascribe to steps taken towards the origin. Anomalous diffusion is therefore achieved by the consequences of the step direction: The walker faces an enhanced probability of detachment only if its step is towards the origin. Thus, the trajectories that tend towards the origin are filtered out as time progresses, thereby splitting the central mode into two symmetric modes about the origin (Fig. 2). Despite the asymmetry being applied at $t_{0}$, the MSD may report an anomalously diffusive $\alpha$ only at long time lags if $k_{\mathrm{d}}$ is small. Similarly, Fig. 10 shows that for small-to-intermediate time lags, $\gamma_{2}$ can fluctuate about the Gaussian value of unity before systematically deviating from it. Therefore, we suspect the effects of biased detachment on the MSD may be difficult to deconvolve given limited statistics for systems with a small $k_{\mathrm{d}}$.

\section{CONCLUSIONS}

The MSD is commonly used to assess anomalous diffusion in microscopic systems. Here, we assessed the effect of a biased detachment probability on the MSD of one-dimensional random walkers, employing both constant and exponential detachment probabilities. We found that the detachment rate controls the apparent dynamics of the system: More gradual detachment (smaller $k_{\mathrm{d}}$ ) delays the onset of superballistic behavior, but results in a larger and longer-lasting excursion into the superballistic regime [Fig. 2(d)]. All of these biaseddetachment systems eventually relax to the ballistic limit $(\alpha=2)$. Figure 5 shows a comparison between the trajectory and ensemble approaches where the superballistic behavior is most clearly seen in the (much longer-time) ensemblelevel model. The limited statistics arising from detachment of trajectory-resolved systems means they cannot easily provide measures of long-time superballistic behaviors; however, the statistics are sufficient to demonstrate a strong superdiffusive bias.

The detachment bias confounds our ability to infer dynamical properties: The MSD suggests highly anomalous behavior, while the underlying dynamics we have imposed are Brownian. Therefore, for systems with trajectories of varying duration, a superdiffusive MSD should not on its own be taken to demonstrate directionality of a walker; such insight would require deconvolving the effects of processivity.

It has previously been shown that a misinterpretation of the MSD can arise in single-particle tracking experiments due to measurement error and inherent system heterogeneity [18]. Researchers have also cautioned about overinterpretation of the MSD for continuous-time random walks with powerlaw waiting-time distributions, where improper averaging can lead to false conclusions about transport properties [49]. We further caution the use of the MSD for systems with finite processivity, where detachment bias may lead to an overestimate of the anomalous diffusion exponent.

\section{ACKNOWLEDGMENTS}

This work was funded by the Natural Sciences and Engineering Research Council of Canada (NSERC) through Discovery Grants to N.R.F. and D.A.S. and a Postgraduate Scholarship-Doctoral to C.S.K., and by a Tier-II Canada Research Chair to D.A.S. Computational resources were provided by Compute Canada.

\section{APPENDIX A: ANALYTICAL EXPRESSION FOR DIFFUSION EXPONENT $\alpha$}

The ensemble-averaged mean-squared displacement is defined as

$$
\left\langle[r(n)-r(0)]^{2}\right\rangle=\frac{1}{N} \sum_{i=1}^{N}\left[r_{i}(n)-r_{i}(0)\right]^{2}=D_{\mathrm{g}} n^{\alpha} .
$$

Here, $n$ is an arbitrary point in time, $N$ is the size of the ensemble, $D_{\mathrm{g}}$ is the general diffusion coefficient, and $\alpha$ is the anomalous diffusion exponent. We can also consider a point $n-b$ somewhat earlier in time,

$$
\left\langle[r(n-b)-r(0)]^{2}\right\rangle=D_{\mathrm{g}}(n-b)^{\alpha} .
$$

The ratio of (A1) and (A2) gives

$$
\begin{gathered}
\frac{\left\langle[r(n)-r(0)]^{2}\right\rangle}{\left\langle[r(n-b)-r(0)]^{2}\right\rangle}=\frac{n^{\alpha}}{(n-b)^{\alpha}}, \\
\log _{n} \frac{\left\langle[r(n)-r(0)]^{2}\right\rangle}{\left\langle[r(n-b)-r(0)]^{2}\right\rangle}=\log _{n} \frac{n^{\alpha}}{(n-b)^{\alpha}} \\
=\alpha \log _{n} n \\
-\log _{n}\left[(n-b)^{\alpha}\right] .
\end{gathered}
$$


Using the logarithmic identity

$$
\log _{b} x=\frac{\log _{d} x}{\log _{d} b}
$$

we then solve for $\alpha$,

$$
\begin{gathered}
\log _{n} \frac{\left\langle[r(n)-r(0)]^{2}\right\rangle}{\left\langle[r(n-b)-r(0)]^{2}\right\rangle}=\alpha\left(1-\frac{1}{\log _{n-b} n}\right), \\
\alpha_{n-b}^{n}=\left(1-\frac{1}{\log _{n-b} n}\right)^{-1} \log _{n} \frac{\left\langle[r(n)-r(0)]^{2}\right\rangle}{\left\langle[r(n-b)-r(0)]^{2}\right\rangle} .
\end{gathered}
$$

The $\alpha_{n-b}^{n}$ notation denotes that $\alpha$ is estimated by two points, at times $n$ and $n-b$. Equation (A8) is an expression for $\alpha$ derived from an arbitrary ensemble-averaged mean-squared displacement. In the work presented in this paper we restrict ourselves to one dimension and have a system for which $\langle x\rangle=0$. Equation (A8) then reduces to

$$
\alpha_{n-b}^{n}=\left(1-\frac{1}{\log _{n-b} n}\right)^{-1} \log _{n} \frac{\left\langle x^{2}(n)\right\rangle}{\left\langle x^{2}(n-b)\right\rangle} .
$$

In this work we apply (A9) with $b=2$ to analytical ensemble-level results, to estimate the anomalous diffusion exponent over a duration of two time steps.

\section{APPENDIX B: COMPARING THE DYNAMIC FUNCTIONAL TO NEWLY DERIVED $\boldsymbol{\alpha}_{n-b}^{n}$}

To demonstrate the utility of $\alpha_{n-b}^{n}$ (6), we determine the anomalous diffusion coefficient for an example in which biased detachment is suddenly turned on after 100 time steps. Figure 8(a) shows conventional diffusion for the first 100 time steps, after which there is a sudden increase in the $\mathrm{MSD}_{\mathrm{EA}}$. Figure 8(b) compares $\alpha$ determined using (6) and (5). The derivative used to calculate $\alpha_{\psi}$ via (5) is a smoothing spline derivative with cross-validation [50], while the expression (6) for $\alpha_{n-b}^{n}$ is exact.
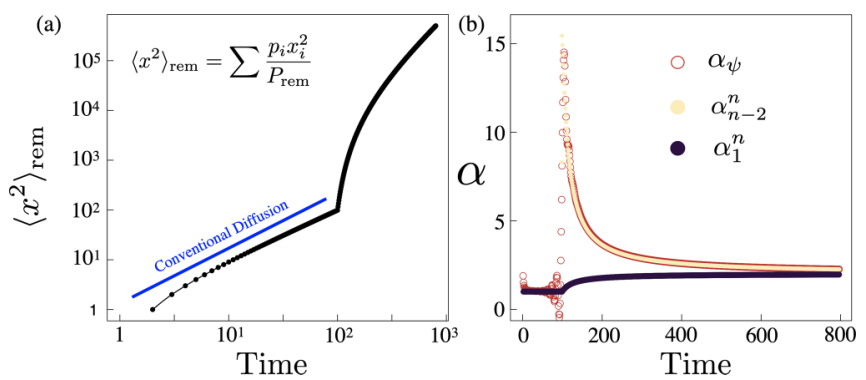

FIG. 8. (a) An example of a $\log -\log \mathrm{MSD}_{\mathrm{EA}}$-time trend from the analytical model where detachment is turned on at $t=100$. (b) A comparison of $\alpha$ computed numerically by (5) and analytically by (6).
TABLE II. $\left\langle x^{2}\right\rangle_{\text {rem }}$ and $\alpha_{n-2}^{n}$ for the first four time steps.

\begin{tabular}{lcccc}
\hline \hline Time step & $P_{\text {rem }}$ & $\sum p_{i} x_{i}^{2}$ & $\left\langle x^{2}\right\rangle_{\text {rem }}$ & $\alpha_{n-2}^{n}$ \\
\hline 0 & 1 & 0 & 0 & \\
1 & 1 & 1 & 1 & \\
2 & $\frac{1}{2}(1+r)$ & 2 & $\frac{4}{1+r}$ & \\
3 & $\frac{1}{4}(1+3 r)$ & $\frac{3}{4}(3+r)$ & $\frac{9+3 r}{1+3 r}$ & $\log _{3} \frac{9+3 r}{1+3 r}$ \\
4 & $\frac{1}{8}+\frac{1}{2} r+\frac{3}{8} r^{2}$ & $2(1+r)$ & $\frac{16}{1+3 r}$ & $2 \log _{4} \frac{4 r+1)}{3 r+1}$ \\
\hline \hline
\end{tabular}

\section{APPENDIX C: ANALYTICAL CALCULATIONS FOR THE DISCRETE RANDOM WALK WITH DETACHMENT}

Table II provides sample analytical calculations from Table I of the ensemble-level $\operatorname{MSD}_{\mathrm{EA}}$ (4) and the analytical anomalous diffusion exponent $\alpha_{n-2}^{n}$ derived in Appendix A, up to four time steps. Here, $d$ is the probability of detachment (3), and $r \equiv 1-d$ represents the probability of remaining attached to the lattice for an individual step. $P_{\text {rem }}$ is a normalization factor and represents the total probability of remaining attached to the lattice.

\section{APPENDIX D: DERIVATION OF $\left\langle v_{+}\right\rangle$FOR THE ENSEMBLE-LEVEL MODEL}

Here, we derive a general expression for the ensemble level $\left\langle v_{+}\right\rangle$, the mean velocity conditioned on the walker being at positive displacement. We consider a discrete system in one dimension that can take steps to the left or right with step size $|\Delta x|=1$ and $\Delta t=1$. The probability of stepping left or right is equal. Let $d_{1}(t)$ and $d_{\mathrm{r}}(t)$ represent the probability of detaching from the lattice for steps taken to the left and right, respectively. We then write the normalized probability at time $t$ of taking a step to the left and remaining attached as

$$
P\left(\Delta x_{\mathrm{left}}\right)=\frac{1-d_{1}(t)}{2-d_{1}(t)-d_{\mathrm{r}}(t)},
$$

and the normalized probability of taking a step to the right and remaining attached as

$$
P\left(\Delta x_{\text {right }}\right)=\frac{1-d_{\mathrm{r}}(t)}{2-d_{1}(t)-d_{\mathrm{r}}(t)} .
$$

Thus, the average displacement after one time step is $\langle\delta x\rangle=$ $\left[P\left(\Delta x_{\text {right }}\right)-P\left(\Delta x_{\text {left }}\right)\right] \Delta x$. Then

$$
\left\langle v_{+}\right\rangle=\frac{\langle\delta x\rangle}{\Delta t}=\frac{d_{1}(t)-d_{\mathrm{r}}(t)}{2-d_{1}(t)-d_{\mathrm{r}}(t)} \frac{\Delta x}{\Delta t} .
$$

The effects of detachment on the mean ensemble acceleration $\left\langle a_{+}\right\rangle=\frac{d}{d t}\left\langle v_{+}\right\rangle$can then be determined, as can the conditional mean displacement, $\left\langle x_{+}\right\rangle=\int d t\left\langle v_{+}\right\rangle$.

In this work we consider exponential detachment for particles moving towards the origin, $d_{\mathrm{r}}(t)=0$ and $d_{1}(t)=d(t)=$ $1-e^{-k_{\mathrm{d}} t}$. We then have

$$
\left\langle v_{+}\right\rangle_{\exp }=\frac{1-e^{-k_{\mathrm{d}} t}}{1+e^{-k_{\mathrm{d}} t}} .
$$

We also consider the case where detachment towards the origin is constant with time, $d_{\mathrm{r}}(t)=0$ and $d_{1}(t)=d$, 


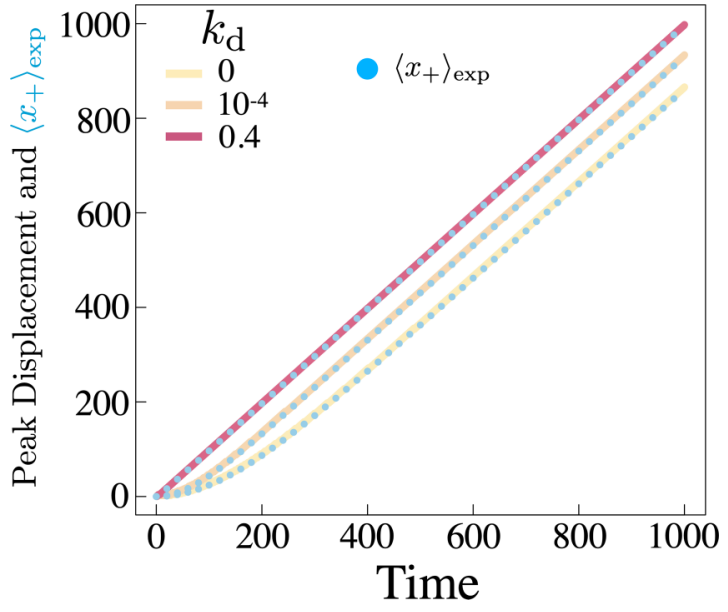

FIG. 9. Conditional mean $\left\langle x_{+}\right\rangle_{\exp }$ (D6) (blue dots) from theory agrees with ensemble-level positive peak displacement (curves) from Fig. 3(c).

giving

$$
\left\langle v_{+}\right\rangle_{\text {const }}=\frac{d}{2-d} .
$$

To demonstrate agreement of this approach with our numerical data, we compare for the exponential case the positive peak displacement obtained from the ensemble-level simulations with the analytical conditional mean,

$$
\begin{aligned}
& \left\langle x_{+}\right\rangle_{\exp }=\int d t \frac{1-e^{-k_{\mathrm{d}} t}}{1+e^{-k_{\mathrm{d}} t}} \\
= & \frac{2 \log \left(e^{k_{\mathrm{d}} t}+1\right)}{k_{\mathrm{d}}}-t+C,
\end{aligned}
$$

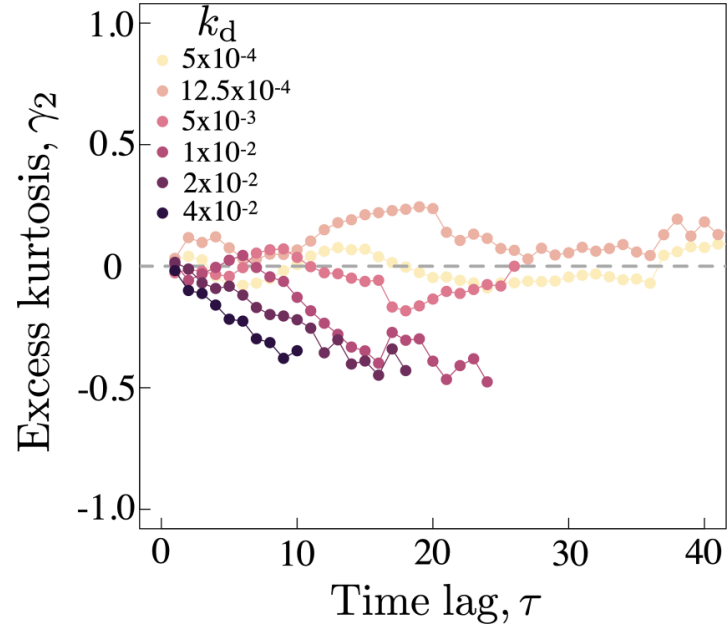

FIG. 10. Excess kurtosis as a function of time lag for the trajectory-resolved model.

where $C$ is an integration constant. We set the integration constant to $C=-\frac{2 \log 2}{k_{\mathrm{d}}}$ such that $\left\langle x_{+}\right\rangle_{\exp }(t=0)=0$. We find this analytical expression for $\left\langle x_{+}\right\rangle_{\exp }$ to agree with the ensemblelevel results for exponential detachment (Fig. 9).

\section{APPENDIX E: FURTHER KURTOSIS ANALYSIS OF TRAJECTORY-RESOLVED MODEL}

In Fig. 10, we plot the excess kurtosis as a function of time lag for various $k_{\mathrm{d}}$ of the trajectory-resolved simulations. For small $k_{\mathrm{d}}, \gamma_{2}$ tends to fluctuate about the Gaussian value of unity. For larger $k_{\mathrm{d}}, \gamma_{2}$ tends to negative values.
[1] A. Einstein, Investigations on the Theory of the Brownian Movement, edited by R. Fürth and A. D. Cowper (Dover, New York, 1956) .

[2] R. Metzler and J. Klafter, The random walk's guide to anomalous diffusion: A fractional dynamics approach, Phys. Rep. 339, 1 (2000).

[3] L. F. Richardson, Atmospheric diffusion shown on a distanceneighbour graph, Proc. R. Soc. London 110, 709 (1926).

[4] S. Havlin and D. Ben-Avraham, Diffusion in disordered media, Adv. Phys. 51, 187 (2002).

[5] R. Metzler, J. H. Jeon, A. G. Cherstvy, and E. Barkai, Anomalous diffusion models and their properties: Non-stationarity, non-ergodicity, and ageing at the centenary of single particle tracking, Phys. Chem. Chem. Phys. 16, 24128 (2014).

[6] J. D. Bao and Y. Z. Zhuo, Ballistic Diffusion Induced by a Thermal Broadband Noise, Phys. Rev. Lett. 91, 138104 (2003).

[7] O. Feinerman, I. Pinkoviezky, A. Gelblum, E. Fonio, and N. S. Gov, The physics of cooperative transport in groups of ants, Nat. Phys. 14, 683 (2018).

[8] M. Rank, L. Reese, and E. Frey, Cooperative effects enhance the transport properties of molecular spider teams, Phys. Rev. E 87, 032706 (2013).

[9] D. ben-Avraham, F. Leyvraz, and S. Redner, Superballistic motion in a "random-walk" shear flow, Phys. Rev. A 45, 2315 (1992).
[10] P. F. C. Tilles, S. V. Petrovskii, and P. L. Natti, A random acceleration model of individual animal movement allowing for diffusive, superdiffusive and superballistic regimes, Sci. Rep. 7, 14364 (2017).

[11] T. Li, S. Kheifets, D. Medellin, and M. G. Raizen, Measurement of the instantaneous velocity of a Brownian particle, Science 328, 1673 (2010).

[12] J. Duplat, S. Kheifets, T. Li, M. G. Raizen, and E. Villermaux, Superdiffusive trajectories in Brownian motion, Phys. Rev. E 87, 020105(R) (2013).

[13] A. G. Cherstvy and R. Metzler, Anomalous diffusion in timefluctuating non-stationary diffusivity landscapes, Phys. Chem. Chem. Phys. 18, 23840 (2016).

[14] F. Le Vot, E. Abad, and S. B. Yuste, Continuous-time randomwalk model for anomalous diffusion in expanding media, Phys. Rev. E 96, 032117 (2017).

[15] H. Salman, Y. Gil, R. Granek, and M. Elbaum, Microtubules, motor proteins, and anomalous mean squared displacements, Chem. Phys. 284, 389 (2002).

[16] J. R. Howse, R. A. L. Jones, A. J. Ryan, T. Gough, R Vafabakhsh, and R. Golestanian, Self-Motile Colloidal Particles: From Directed Propulsion to Random Walk, Phys. Rev. Lett. 99, 048102 (2007).

[17] S. Manley, J. M. Gillette, G. H. Patterson, H. Shroff, H. F. Hess, E. Betzig, and J. Lippincott-Schwartz, High-density mapping 
of single-molecule trajectories with photoactivated localization microscopy, Nat. Methods 5, 155 (2008).

[18] E. Kepten, I. Bronshtein, and Y. Garini, Improved estimation of anomalous diffusion exponents in single-particle tracking experiments, Phys. Rev. E 87, 052713 (2013).

[19] S. K. Ghosh, A. G. Cherstvy, D. S. Grebenkov, and R. Metzler, Anomalous, non-Gaussian tracer diffusion in crowded twodimensional environments, New J. Phys. 18, 013027 (2016).

[20] S. Karmakar, C. Dasgupta, and S. Sastry, Short-Time Beta Relaxation in Glass-Forming Liquids is Cooperative in Nature, Phys. Rev. Lett. 116, 085701 (2016).

[21] D. S. Martin, M. B. Forstner, and J. A. Käs, Apparent subdiffusion inherent to single particle tracking, Biophys. J. 83, 2109 (2002).

[22] T. Savin, Microrheology of heterogeneous systems, Massachusetts Institute of Technology: MIT OpenCourseWare, https://ocw.mit.edu/.

[23] L. van Hove, Correlations in space and time and Born approximation scattering in systems of interacting particles, Phys. Rev. 95, 249 (1954).

[24] A. Z. Akcasu, N. Corngold, and J. J. Duderstadt, Theory of self-diffusion in classical fluids: The Van Hove self-correlation function $G_{s}(r, t)$, Phys. Fluids 13, 2213 (1970).

[25] A. G. Cherstvy, S. Thapa, C. E. Wagner, and R. Metzler, NonGaussian, non-ergodic, and non-Fickian diffusion of tracers in mucin hydrogels, Soft Matter 15, 2526 (2019).

[26] A. V. Weigel, B. Simon, M. M. Tamkun, and D. Krapf, Ergodic and nonergodic processes coexist in the plasma membrane as observed by single-molecule tracking, Proc. Natl. Acad. Sci. USA 108, 6438 (2011).

[27] G. Bel and E. Barkai, Weak Ergodicity Breaking in the Continuous-Time Random Walk, Phys. Rev. Lett. 94, 240602 (2005).

[28] N. B. R. Orsini and S. Greenland, Generalized least squares for trend estimation of summarized dose-response data, Stata J. 6, 40 (2009).

[29] A. Rahman, Correlations in the motion of atoms in liquid Argon, Phys. Rev. 136, A405 (1964).

[30] W. Kob and H. C. Andersen, Testing made-coupling theory for a supercooled binary Lennard-Jones mixture: The van Hove correlation function, Phys. Rev. E 51, 4626 (1995).

[31] M. Richard, C. Blanch-Mercader, H. Ennomani, W. Cao, and M. Enrique, Active cargo positioning in antiparallel transport networks, Proc. Natl. Acad. Sci. USA 116, 14835 (2019).

[32] R. Pei, S. K. Taylor, D. Stefanovic, S. Rudchenko, T. E. Mitchell, and M. N. Stojanovic, Behavior of polycatalytic assemblies in a substrate-displaying matrix, J. Am. Chem. Soc. 128, 12693 (2006).

[33] K. Lund, A. J. Manzo, N. Dabby, N. Michelotti, A. JohnsonBuck, J. Nangreave, S. Taylor, R. Pei, M. N. Stojanovic, N. G. Walter, E. Winfree, and H. Yan, Molecular robots guided by prescriptive landscapes, Nature (London) 465, 206 (2010).

[34] S. Kovacic, L. Samii, P. M. Curmi, H. Linke, M. J. Zuckermann, and N. R. Forde, Design and construction of the Lawnmower, an artificial burnt-bridges motor, IEEE Trans. Nanobiosci. 14, 305 (2015).

[35] K. Yehl, A. Mugler, S. Vivek, Y. Liu, Y. Zhang, M. Fan, E. R. Weeks, and K. Salaita, High-speed DNA-based rolling motors powered by RNase H, Nat. Nanotechnol. 11, 184 (2015).

[36] A. T. Blanchard, A. S. Bazrafshan, J. Yi, J. T. Eisman, K. M. Yehl, T. Bian, A. Mugler, and K. Salaita, Highly polyvalent DNA motors generate $100+\mathrm{pN}$ of force via autochemophoresis, Nano Lett. 19, 6977 (2019).

[37] A. Bazrafshan, T. Meyer A, H. Su, J. Brockman M, A Blanchard T, S. Pirane, Y. Duan, Y. Ke, and K. Salaita, Tunable DNA origami motors translocate ballistically over $\mu$ m distances at nm/s speeds, Angew. Chem., Int. Ed. 59, 9514 (2020).

[38] L. Samii, H. Linke, M. J. Zuckermann, and N. R. Forde, Biased motion and molecular motor properties of bipedal spiders, Phys. Rev. E 81, 021106 (2010).

[39] L. Samii, G. A. Blab, E. H. C. Bromley, H. Linke, P. M. G. Curmi, M. J. Zuckermann, and N. R. Forde, Time-dependent motor properties of multipedal molecular spiders, Phys. Rev. E 84, 031111 (2011).

[40] M. J. Olah and D. Stefanovic, Superdiffusive transport by multivalent molecular walkers moving under load, Phys. Rev. E 87, 062713 (2013).

[41] C. S. Korosec, M. J. Zuckermann, and N. R. Forde, Dimensionality-dependent crossover in motility of polyvalent burnt-bridges ratchets, Phys. Rev. E 98, 032114 (2018).

[42] C. S. Peskin, G. M. Odell, and G. F. Oster, Cellular motions and thermal fluctuations: The Brownian ratchet, Biophys. J. 65, 316 (1993).

[43] N. Gal and D. Weihs, Experimental evidence of strong anomalous diffusion in living cells, Phys. Rev. E 81, 020903(R) (2010).

[44] Y. Inoue, A. H. Iwane, T. Miyai, E. Muto, and T. Yanagida, Motility of single one-headed kinesin molecules along microtubules, Biophys. J. 81, 2838 (2001).

[45] J. L. Ross, K. Wallace, H. Shuman, Y. E. Goldman, and E. L. F. Holzbaur, Processive bidirectional motion of dynein-dynactin complexes in vitro, Nat. Cell Biol. 8, 562 (2006).

[46] M. Brunstein, L. Bruno, M. Desposito, and V. Levi, Anomalous dynamics of melanosomes driven by myosin- $\mathrm{V}$ in xenopus laevis melanophores, Biophys. J. 97, 1548 (2009).

[47] G. D. Joseph Phillies, In complex fluids the Gaussian Diffusion Approximation is generally invalid, Soft Matter 11, 580 (2015).

[48] B. Wang, J. Kuo, S. C. Bae, and S. Granick, When Brownian diffusion is not Gaussian, Nat. Mater. 11, 481 (2012).

[49] A. Lubelski, I. M. Sokolov, and J. Klafter, Nonergodicity Mimics Inhomogeneity in Single Particle Tracking, Phys. Rev. Lett. 100, 250602 (2008).

[50] G. Wahba, Bayesian "Confidence intervals" for the crossvalidated smoothing spline, J. R. Stat. Soc. B 45, 133 (1983).

Correction: The previously published Figure 1(c) was processed incorrectly during production, causing overlapping of axis labels. The figure has now been set properly. 